\title{
Santiago de Chile: ¿ciudad de ciudades? Desigualdades sociales en zonas de mercado laboral local ${ }^{1}$
}

\author{
Luis Fuentes, Oscar Mac-Clure, Cristóbal Moya \\ y Camilo Olivos
}

\section{Resumen}

El propósito del presente artículo es delimitar y caracterizar la estructura urbana de Santiago de Chile a partir de la relación entre los lugares de residencia y de trabajo de sus habitantes, que conforman zonas de mercado laboral local. Se exponen los criterios y procedimientos metodológicos empleados, de acuerdo con los cuales se establecen estas zonas y se las describe sobre la base de esta delimitación funcional, a fin de determinar en qué medida condicionan las desigualdades sociales en la ciudad. Se analizan en términos espaciales la desigualdad de ingresos, el acceso a la educación y la composición de las clases sociales.

Palabras clave

Ciudades, mercado de trabajo, empleo, domicilio y residencia, clases sociales, igualdad, ingresos, educación, indicadores socioeconómicos, Chile

Clasificación JEL

J60, D63, Z13

Autores

Luis Fuentes es Académico del Instituto de Estudios Urbanos y Territoriales e Investigador del Centro de Desarrollo Urbano Sustentable (CEDEUS) de la Pontificia Universidad Católica de Chile.Ifuentes@uc.cl

Oscar Mac-Clure es Académico del Centro de Estudios del Desarrollo Regional y Políticas Públicas (CEDER) de la Universidad de Los Lagos, Chile. oscar.macclure@gmail.com

Cristóbal Moya es Docente de la Escuela de Sociología de la Universidad Diego Portales, Chile.cristobalmoya@gmail.com

Camilo Olivos es Asistente de Investigación en el Instituto de Estudios Urbanos y Territoriales de la Pontificia Universidad Católica de Chile. colivos17@gmail.com

\footnotetext{
Para la elaboración de este artículo se contó con el apoyo del Fondo Nacional de Desarrollo Científico y Tecnológico de Chile (FONDECYT), por intermedio de los proyectos 1141157 y 1150808, y de la Comisión Nacional de Investigación Científica y Tecnológica (CONICYT), mediante el proyecto CONICYT/FONDAP 15110020.
} 


\section{Introducción}

La globalización ha dado origen a diversas transformaciones de la geografía económica mundial y, en este marco, las ciudades han actuado como articuladoras de la internacionalización de las economías (Sassen, 2001). Las ciudades de América Latina no han estado ajenas a este proceso (De Mattos, 2010), en el que Santiago de Chile representa un caso especial, debido a la temprana inserción de la economía nacional en la global, y a los consecuentes cambios en la función, morfología y estructura socioespacial de la ciudad a lo largo de diferentes períodos.

Las tendencias recientes que muestra el crecimiento urbano de Santiago han añadido complejidad a su estructura, en virtud de procesos que han sido objeto de estudios específicos. Sin embargo, como veremos más adelante, se debería avanzar aún más en la comprensión de las modalidades de funcionamiento, las características generales y las desigualdades que presenta la urbe. Al respecto, las preguntas que abordamos se refieren a la configuración espacial de la relación entre el lugar de residencia y de trabajo. Específicamente ¿cuál es la estructura funcional de la ciudad?, y ¿en qué medida se vincula con las desigualdades urbanas y conforme a qué mecanismos? Se trata, concretamente, de identificar las posibilidades de caracterizar y analizar la evolución de la estructura funcional de la ciudad y las tendencias que explican las desigualdades urbanas en los últimos años.

A partir de las conclusiones de distintos estudios y el análisis de las variadas dimensiones de la evolución urbana, se intenta contribuir al debate sobre la estructura de Santiago, en función de una clasificación de los espacios sociales y conforme a criterios relacionados con el lugar de trabajo y de residencia, a fin de delimitar la ciudad a una escala agregada. Asimismo, se identifican las zonas de mercado laboral definidas por la relación entre la oferta y la demanda de trabajo en el espacio de la ciudad. Para validar esta metodología, se somete a prueba su aporte a la caracterización de la urbe desde la perspectiva de las desigualdades sociales en términos de ingresos, educación, clases y otros indicadores pertinentes.

Postulamos que una configuración espacial basada en la relación entre residencia y trabajo ofrece una imagen de conjunto de la estructura y el funcionamiento de una gran ciudad como Santiago, y presenta la ventaja de contribuir a la comprensión de las desigualdades sociales que se dan en ella. Esto se debe a que cada una de las zonas consideradas presenta características particulares en términos de los indicadores socioeconómicos básicos, el nivel y la distribución del ingreso, y la composición de las clases sociales. Asimismo, demostramos que las desigualdades socioeconómicas son acentuadas en algunas de las zonas y que también hay grandes diferencias entre algunas de ellas. Por suponer que existe una relación bidireccional entre la configuración espacial y las desigualdades urbanas, en el enfoque empleado se otorga prioridad a la influencia del espacio social en estas últimas.

En la sección II del artículo se describe el debate académico en torno a la estructura de la ciudad y el enfoque metodológico centrado en las zonas de mercado laboral local. En la sección III se expone en detalle el método aplicado en este estudio para la delimitación e identificación de estas zonas en Santiago. En la sección IV se analiza la conformación de las zonas de mercado laboral y se caracterizan las desigualdades socioeconómicas y su composición social. Finalmente, se analizan las conclusiones relativas a la configuración espacial de la ciudad y a su capacidad de agregar valor al análisis de las desigualdades sociales intraurbanas. 


\section{Marco general de los espacios urbanos}

\section{Estudios de la estructura territorial de Santiago}

En el presente artículo se propone considerar los mercados de trabajo existentes en Santiago como un factor clave para comprender su heterogeneidad. En varios estudios sobre esta y otras ciudades de América Latina, se ha hecho hincapié en la creciente complejidad estructural derivada de los nuevos procesos de crecimiento urbano. En un estudio de Dockendorff y otros (1990), en el que se describe la estructura socioeconómica y espacial del Gran Santiago, se concluyó que el rasgo distintivo que aparecía como una constante era la segmentación social intercomunal.

En estudios más recientes se ha prestado particular atención a diversas dinámicas urbanas, entre otras las trayectorias residenciales y la migración interna (De Mattos, Fuentes y Link, 2014; Ortiz y Escolano, 2013; Ortiz y Morales, 2002); los recientes fenómenos de reubicación de centros comerciales y empleos (Escolano y Ortiz, 2005; Rodríguez, 2012; Truffello e Hidalgo, 2015); la transformación de los patrones de segregación residencial (Agostini, 2010; Sabatini, Cáceres y Cerda, 2001), y la nueva morfología generada por el sistema financiero en el sector de la vivienda (Cattaneo, 2012).

En algunos de los estudios mencionados se ha intentado clasificar y caracterizar distintas zonas funcionales de Santiago, mediante un análisis de la policentralidad desde el punto de vista de la densidad comercial y la movilidad. Truffello e Hidalgo (2015) observan que Santiago se caracteriza por una estructura policéntrica del comercio, aunque también presenta rasgos monocéntricos, ya que su centro de negocios sigue teniendo un rol predominante. Tomando en consideración variables como la distribución de los empleos y los núcleos comerciales ("centralidades"), Rodríguez (2012) concluye que coexisten un incipiente proceso de aparición de "subcentralidades" y la persistencia del centro comercial histórico, lo que acentúa la relevancia del estudio de la dimensión espacial del mercado laboral. Al respecto, es difícil encontrar análisis de la configuración territorial de los mercados de trabajo de Santiago, que vayan más allá de los estudios generales sobre la metrópolis (De Mattos, 2002; Pollack y Uthoff, 1987; Riffo, 2004) en los que se vinculan sus características o su evolución a la estratificación social, la pobreza y la segregación residencial.

En cuanto a las desigualdades socioeconómicas que se registran en Santiago, Agostini (2010) señala que, pese al mejoramiento de las condiciones de vida en los últimos años, hay acentuadas diferencias de ingreso entre sus habitantes y los residentes de comunas del resto del país. A partir de un examen de los coeficientes de Gini comunales, este autor estudia la heterogeneidad y la homogeneidad de ingresos en las comunas más ricas y más pobres, y concluye que es errada la percepción generalizada de que las primeras son más homogéneas y las segundas más heterogéneas.

Por otra parte, Arriagada (2010) sostiene que la segregación se intensificó en Chile como consecuencia de la aplicación de la política de vivienda social en algunas áreas de la ciudad y de la concentración de infraestructura urbana en zonas acomodadas del sector oriente, lo que se suma a la expansión vertiginosa hacia barrios periféricos con insuficiente equipamiento.

La segmentación interna ha sido analizada por Sabatini, Cáceres y Cerda (2001), que definen el cono oriente de Santiago como un área heterogénea desde el punto de vista social y las periferias de otros sectores de la ciudad como lugares estigmatizados en los que se acumulan problemas sociales. Tomando en consideración múltiples variables, entre las que destacan el ingreso y la educación, se ha descrito la conformación de zonas en las que se manifiesta la segmentación social existente (Heinrichs y otros, 2011).

Con el propósito de realizar estudios técnicos sobre el sistema de transporte urbano, la Secretaría de Planificación de Transporte ha dividido la ciudad en macrozonas, entre las que se 
producen desplazamientos que se cuantifican en las encuestas de origen y destino (SECTRA, 1992). Además, en un estudio sobre la distribución del poder y la gobernabilidad (Orellana y Fuentes, 2007) también se desarrolló una clasificación de las comunas de Santiago.

En el examen de la literatura, se ha constatado que consiste en estudios centrados en temas específicos, como el desplazamiento por motivos de trabajo y la transición de la monocentralidad a la policentralidad, o en problemas acotados, como la segregación y la falta de gobernabilidad de la ciudad. Tomando como punto de partida las conclusiones de los análisis de esos aspectos de la evolución urbana, con el presente artículo se intenta hacer un aporte a los estudios anteriores, como parte de un debate sobre la estructura urbana de Santiago en su conjunto. A nuestro juicio, antes de proceder a una caracterización socioeconómica o en función de otras variables, la distinción básica de zonas basada en los mercados del trabajo locales facilita la comprensión de las desigualdades sociales que se registran en la ciudad y que, en última instancia, han sido el tema central de la mayor parte de los estudios.

\section{Estudios de zonas de mercado laboral local}

El propósito fundamental de este artículo es contribuir al debate sobre la dimensión territorial del mercado laboral, mediante un análisis de los traslados por razones de trabajo similar al aplicado en investigaciones realizadas en los Estados Unidos (Singelmann y Deseran, 1993; Tolbert y Sizer, 1996), en España (Casado-Díaz, 2003), en Alemania (Russo y otros, 2011) y en otros países (Casado-Díaz y Coombes, 2011; OCDE, 2002)2. Estos espacios se han conceptualizado de diversas maneras. En Gran Bretaña se estudiaron las áreas dentro de las cuales los trabajadores deben desplazarse por motivos de trabajo (Coombes y Bond, 2008); en Italia, los sistemas de trabajo local (ISTAT, 2005), y en Francia, las "cuencas de vida" y los territorios habitados (Vallès, 2004). Es importante que exista una agenda de investigación comparativa sobre las zonas de mercado laboral a nivel internacional, dado que una de las dificultades que se presentaban hace algunas décadas para el proceso de regionalización era la definición de los territorios más relevantes para las políticas públicas nacionales. Asimismo, para responder más eficazmente a la globalización de la economía y la sociedad, conviene establecer áreas comparables entre países (Casado-Díaz y Coombes, 2011).

En uno de las primeros estudios en los que se consideró la dimensión territorial de los mercados de trabajo, realizado en los Estados Unidos en 1940, se planteaba la existencia de zonas en las que los trabajadores podían cambiar de trabajo sin cambiar de residencia (Casado-Díaz y Coombes, 2011). Teniendo como base esos primeros estudios, se comenzó a definir este concepto como el área que abarca tanto el lugar de residencia como el de trabajo de una determinada población local (Tolbert y Sizer, 1996). Así, en la mayoría de las definiciones actuales de las zonas de mercado laboral se otorga particular importancia a la relación entre el lugar de residencia y de trabajo, así como a su vinculación mediante traslados cotidianos. Desde el punto de vista espacial, esto ha conducido a la delimitación de zonas de acuerdo con dos criterios claves: el punto de encuentro de la oferta y la demanda de empleo, y el área que define la interacción entre el lugar de residencia y el de trabajo. De acuerdo con estos criterios, se examina la cercanía entre varios municipios ${ }^{3}$, lo que supone un nuevo análisis y, a la larga, la adopción de nuevas divisiones administrativas que reemplazan a las tradicionales.

Uno de los aspectos más destacados de las investigaciones sobre los mercados de trabajo locales realizadas en América Latina, a las que se suma el presente artículo, es su contribución al

2 La metodología utilizada en diversos países y estudios para delimitar zonas de mercado laboral local es, en general, similar a la aplicada en este estudio. Casado-Díaz y Coombes (2011) presentan una completa descripción de los principales procedimientos empleados para efectuar esa delimitación.

3 Como unidades de análisis, también se pueden considerar áreas de menor tamaño, lo que depende de la disponibilidad de datos estadísticos. 
estudio de las desigualdades sociales y espaciales. En el caso de la zona metropolitana del Valle de México, se ha dado cuenta de la complejidad de la estructura urbana, producto de los procesos de crecimiento vinculados a la movilidad de las empresas y los individuos (Casado, 2012). Al igual que en el presente artículo, en el estudio de Casado se examina el interior de la ciudad, con el fin de analizar la estructura urbana a partir de la configuración geográfica de las relaciones económicas y sociales básicas. La zona metropolitana del Valle de México se divide en 12 áreas de mercado laboral local más o menos autónomas en términos de la relación entre residencia y trabajo, pero que presentan una notable concentración del empleo en el distrito de negocios, cuya extensión es más amplia que los límites tradicionales del centro de la ciudad.

La aplicación de una metodología similar en Chile ha contribuido al estudio de nuevas dinámicas en el mundo rural y de inequidades que afectan a distintos tipos de territorios rurales (Berdegué y otros, 2011). Esta metodología se ha utilizado también para determinar si existe una red de ciudades intermedias en el sur del país (Maturana y Arenas, 2012). Junto con distinguir zonas de mercado de trabajo en Chile (Mac-Clure y Calvo, 2013), se las ha vinculado con la distribución del ingreso y la composición de las clases sociales en diversos territorios del país (Mac-Clure, Barozet y Maturana, 2014). El presente artículo se centra en el examen de la ciudad de Santiago de Chile, caracterizada por acentuadas desigualdades, al igual que las demás grandes ciudades de América Latina.

\section{Metodología: delimitación de zonas de mercado laboral local}

Para delimitar las zonas de mercado laboral local en la capital de Chile, se consideran el lugar de residencia y el traslado al trabajo, como se ha hecho en los estudios reseñados con anterioridad.

El método aplicado es de carácter inductivo, lo que significa que el proceso de investigación no se basa en el supuesto de que existe una determinada estructura en torno a la cual se conforman los mercados de trabajo locales, sino que apunta a su identificación. En términos estadísticos, se realizó un examen de conglomerados jerárquicos a partir de las conclusiones del análisis de matrices, con el propósito de integrar los elementos - en este caso, las comunas - en grupos homogéneos, no conocidos de antemano, sino derivados de los datos disponibles. Cabe señalar que en el análisis de conglomerados se emplea un conjunto de métodos y técnicas estadísticas que permiten describir y reconocer las agrupaciones implícitas en un conjunto de datos, por lo que es posible clasificar o dividir en grupos más o menos homogéneos una serie de elementos definidos por diferentes variables.

El universo de este estudio está constituido por 38 comunas de la ciudad de Santiago, que abarcan la Provincia de Santiago y las más importantes de las provincias Cordillera, Chacabuco y Maipo ${ }^{4}$. La unidad mínima de análisis utilizada es la comuna, dado que esto posibilita los análisis socioeconómicos posteriores. La delimitación de las zonas de mercado laboral local se basa en datos de la Encuesta Origen y Destino de Viajes, efectuada por la Secretaría de Planificación de Transporte (SECTRA), con el fin de realizar un diagnóstico del sistema de transporte urbano y planificarlo, dado que contiene datos actualizados sobre traslados 5 . Además, se recurrió a otras fuentes de datos, para validar las zonas identificadas tomando en cuenta otros años ${ }^{6}$.

\footnotetext{
4 Se consideraron las comunas incluidas en los estudios de la Secretaría de Planificación de Transporte (SECTRA).

5 La muestra de la Encuesta Origen y Destino de Viajes de 2012 estuvo integrada por 11.000 hogares, en los que se realizaron entrevistas en días laborables.

6 Se examinaron los resultados de la Encuesta de Caracterización Socioeconómica Nacional (CASEN) de 2009, la única de la serie en que se incluye información sobre traslados cotidianos con motivo de trabajo, y los datos del censo del año 2002.
} 
El proceso de análisis realizado a partir de esa información se dividió en cuatro etapas, en las que se delimitaron las zonas de mercado laboral local (véase el diagrama 1).

Diagrama 1

Secuencia de delimitación de las zonas de mercado laboral local

\begin{tabular}{|c|c|c|c|}
\hline 1 & 2 & 3 & 4 \\
\hline $\begin{array}{l}\text { Construcción } \\
\text { de matriz de } \\
\text { frecuencias } \\
\text { de traslado } \\
\text { entre comunas }\end{array}$ & $\begin{array}{c}\text { Ponderación } \\
\text { del flujo de } \\
\text { traslados }\end{array}$ & $\begin{array}{l}\text { Construcción } \\
\text { de la matriz de } \\
\text { disimilaridad }\end{array}$ & $\begin{array}{c}\text { Análisis de } \\
\text { conglomerados } \\
\text { jerárquicos }\end{array}$ \\
\hline
\end{tabular}

Fuente: Elaboración propia.

a La disimilaridad se refiere al grado de desemejanza entre los datos, sobre la base de la comparación entre una serie y el conjunto.

En primer lugar, se construyó una matriz de doble entrada con las frecuencias de traslado de quienes se desplazan por razones de trabajo entre dos comunas, en cualquier sentido. El segundo paso consistió en la ponderación de las frecuencias; al respecto, es importante tener en cuenta que en el análisis de conglomerados se definen grupos lo más heterogéneos que sea posible; por lo tanto, cuando las variables se expresan en distintas magnitudes, hay que ponderar o estandarizar los datos. El objetivo de la ponderación es evitar sesgos atribuibles al tamaño de las comunas correspondientes a los pares observados. Con tal fin, se corrigen los flujos de traslado, mediante la división del número de personas que se desplazan por razones de trabajo entre dos comunas por la población ocupada de la comuna de menor tamaño. El flujo calculado es un indicador de la intensidad de los traslados por razones de trabajo entre ese par de comunas. Así se desarrolla una matriz de doble entrada, en la que se incluyen las mismas 38 comunas en las columnas y en las líneas.

El tercer paso para la delimitación de las zonas de mercado laboral consiste en aplicar medidas de similitud. En este caso, se elaboró una matriz de distancias o disimilaridad entre las comunas, que ilustra la diferencia entre el valor uno y el flujo de traslado ponderado que se deriva de la matriz anterior. El objetivo de este ejercicio es medir el grado de desemejanza entre los datos, sobre la base de la comparación entre una serie y el conjunto, lo que sirve de base para el posterior desarrollo de una clasificación jerárquica.

En cuarto lugar, se analizan los conglomerados jerárquicos. En este procedimiento se identifican las comunas más próximas en términos de disimilaridad, que se agrupan en un conglomerado. A continuación, se establecen conglomerados cada vez mayores y más heterogéneos, hasta llegar a un único conglomerado global, que corresponde a la ciudad en su conjunto y está integrado por 38 unidades, cada una de las cuales puede considerarse un conglomerado. Esto permite evaluar la heterogeneidad de los conglomerados constituidos en cada etapa y facilita la identificación de grupos homogéneos de comunas, que concuerdan con las conclusiones de los estudios sobre la ciudad. Las comunas agrupadas conforme a los flujos de traslado dentro de un conglomerado se interpretan como mercados laborales locales. En cambio, los conglomerados aislados reflejan un bajo flujo de traslado y se los considera como distintos mercados laborales. Utilizando un dendograma, se hace un corte en uno de los niveles de jerarquía resultantes. Con el propósito analítico de distinguir un número reducido de zonas de mercado laboral en la ciudad, se estableció un umbral de aglomeración en función del flujo de traslado. Este umbral se determinó de conformidad con los criterios especializados sobre la materia, considerando para ello un equilibrio en la conformación de un número reducido de 
zonas de mercado laboral local representativas y manejables estadísticamente ${ }^{7}$. Para corroborar el resultado, se aplicó la técnica estadística de escalamiento multidimensional, que permite desarrollar una representación de las diferencias entre las comunas en lo que respecta al flujo de traslado, lo que facilita la ubicación de las zonas en la ciudad. Luego, para validar las zonas identificadas se analiza si tienen una coherencia geográfica, es decir, si siguen un mismo patrón, mediante diversas fuentes de datos y técnicas estadísticas.

Finalmente, a fin de evaluar la pertinencia y utilidad de la distinción de zonas de mercado laboral para el análisis de las desigualdades sociales existentes en la ciudad, se consideran indicadores socioeconómicos básicos correspondientes a cada una de ellas.

\section{Conformación de las zonas}

\section{Zonas de mercado laboral en Santiago}

A partir de las 38 comunas del Gran Santiago, se configuraron seis conglomerados conforme al método descrito ${ }^{8}$. Sus habitantes se desplazan relativamente menos fuera de ellos por razones de trabajo y los traslados se concentran entre las comunas que los integran. La configuración espacial definida no varía mayormente cuando se emplean distintas fuentes de datos y técnicas de análisis.

A modo ilustrativo, la zona centrooriente es un destino de desplazamientos por motivos de trabajo desde todas las comunas de la ciudad; la frecuencia de traslados entre las comunas que la conforman es alta, en tanto que una proporción relativamente baja de sus residentes viaja a trabajar fuera de ellas. Las zonas resultantes no son espacios cerrados, sino que están integradas en el mercado laboral más amplio de la ciudad, cuyos habitantes se desplazan por razones de trabajo entre múltiples puntos aunque preferentemente dentro de los límites de las zonas identificadas. En el mapa 1 se ilustran las seis zonas delimitadas con la metodología descrita, correspondientes a los mercados de trabajo local de la ciudad de Santiago.

En términos generales, la configuración de las zonas de mercado laboral de Santiago muestra un marcado patrón geográfico, conforme al cual las comunas se agrupan en áreas diferenciadas e independientes. Entre las desviaciones más destacadas del patrón, cabe mencionar los casos de la zona centrooriente, que "se apropia" de la comuna de Santiago, y de la zona sur, un conglomerado de comunas que se proyectan hacia el sector poniente de la ciudad. Por sí solas, estas son conclusiones relevantes, dado que en la mayor parte de los artículos consultados se analiza la configuración de mercados laborales locales en estructuras espaciales agregadas, mientras que en el presente estudio también pretendemos determinar la estructura interna de la ciudad.

Por consiguiente, es interesante comparar estas conclusiones con las expuestas por Casado en su estudio de la zona metropolitana del Valle de México, para el que se empleó una metodología similar (Casado, 2012). El autor identificó una estructura integrada por 12 zonas, que presenta un patrón espacial de tipo radial, diferente al de Santiago; a su vez, este último difiere de las representaciones de la estructura de la ciudad en las que aparece como un centro rodeado de anillos circundantes.

\footnotetext{
7 Se consideraron seis conglomerados de comunas.

8 Las seis zonas abarcan un total de 38 comunas, agrupadas como se indica a continuación. Norte: Colina, Lampa, Renca, Conchalí, Huechuraba, Quilicura, Independencia y Recoleta. Centrooriente: La Reina, Ñuñoa, Providencia, Santiago, Las Condes, Vitacura y Lo Barnechea. Poniente: Cerrillos, Maipú, Estación Central, Lo Prado, Lo Espejo, Cerro Navia, Pudahuel y Quinta Normal. Sur: La Florida, La Granja, San Joaquín, Macul, Peñalolén, Pedro Aguirre Cerda y San Miguel. Suroriente: Puente Alto, La Pintana y Pirque. Surponiente: Calera de Tango, San Bernardo, El Bosque, La Cisterna y San Ramón.
} 
Mapa 1

Santiago de Chile: delimitación y características básicas de las zonas de mercado laboral locala

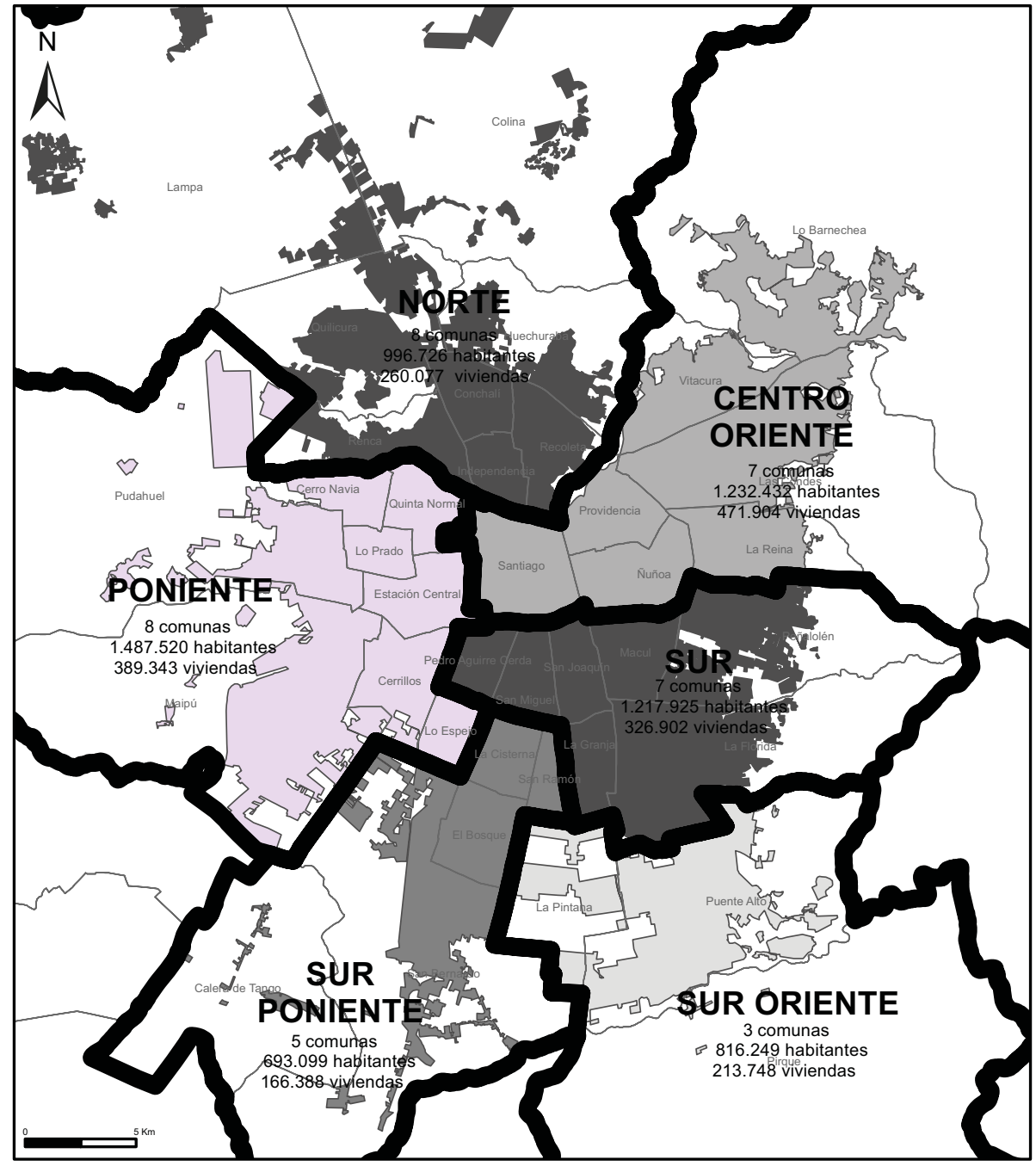

Fuente: Elaboración propia, sobre la base de datos de la Encuesta de Origen y Destino de Viajes de 2012 y de Instituto Nacional de Estadística (INE) de Chile, XVIII Censo de Población de 2012.

a Las zonas indicadas en el mapa corresponden a la denominación utilizada por la Secretaría de Planificación de Transporte.

Esta configuración geográfica es la expresión espacial del análisis del dendograma y del escalamiento multidimensional. En los primeros niveles de aglomeración del dendograma se distinguen la zona centrooriente, la zona sur y la zona suroriente, agrupaciones más homogéneas que las demás cuyos límites son más permeables.

Las zonas no están compuestas por el mismo número de comunas y, por lo tanto, de habitantes y viviendas. Por ejemplo, la zona integrada por menos comunas y habitantes, ubicada en el suroriente de la ciudad, está constituida por Puente Alto, La Pintana y Pirque, que suman más de 800.000 habitantes. En cambio, la zona poniente - la más poblada, y con más comunas y viviendas - abarca ocho comunas en las que viven más de 1.400 .000 personas (véase el mapa 1).

Además de estas diferencias, es importante analizar las zonas de acuerdo con dos conceptos claves: el nivel de autocontención y la porosidad. El primero se refiere al porcentaje de ocupados que 
residen en la misma zona en la que trabajan y de la cual no necesitan salir para trasladarse al lugar de trabajo. El segundo alude a la capacidad de atraer habitantes de otras áreas de mercado laboral por motivos de trabajo. En el cuadro 1 se presenta una síntesis de esta relación.

Cuadro 1

Santiago de Chile: trabajadores residentes en las zonas de mercado laboral, 2009a

(En porcentajes y números absolutos)

\begin{tabular}{|c|c|c|c|c|c|}
\hline \multirow{2}{*}{ Zona de residencia } & \multirow{2}{*}{$\begin{array}{l}\text { Trabajadores que } \\
\text { viven y trabajan en } \\
\text { la misma zona }\end{array}$} & \multicolumn{2}{|c|}{$\begin{array}{l}\text { Personas que se trasladan a trabajar } \\
\text { fuera de la zona de residencia }\end{array}$} & \multicolumn{2}{|c|}{ Total } \\
\hline & & $\begin{array}{l}\text { A la zona } \\
\text { centrooriente }\end{array}$ & A otra zona & $\begin{array}{l}\text { Número de } \\
\text { personas }\end{array}$ & $\begin{array}{l}\text { Porcentaje del } \\
\text { total de la ciudad }\end{array}$ \\
\hline Norte & $56 \%$ & $32 \%$ & $11 \%$ & 373963 & $15,3 \%$ \\
\hline Centrooriente & $81 \%$ & - & $19 \%$ & 582403 & $23,9 \%$ \\
\hline Sur & $35 \%$ & $45 \%$ & $21 \%$ & 447107 & $18,3 \%$ \\
\hline Suroriente & $29 \%$ & $39 \%$ & $32 \%$ & 285155 & $11,7 \%$ \\
\hline Poniente & $45 \%$ & $39 \%$ & $17 \%$ & 510937 & $21,0 \%$ \\
\hline Surponiente & $48 \%$ & $24 \%$ & $29 \%$ & 232882 & $9,5 \%$ \\
\hline Total & & & & 2432449 & $100 \%$ \\
\hline
\end{tabular}

Fuente: Elaboración propia, sobre la base de datos de la Encuesta de Origen y Destino de Viajes de 2012.

a Solo se incluyen los casos sobre los que se dispone de datos sobre traslados.

En la segunda columna de este cuadro se indica el porcentaje de ocupados que trabajan en la misma zona en que residen. Como se puede observar, el mínimo de autocontención es de un 29\%, lo que valida la caracterización de las zonas como mercados laborales endógenos, a pesar de que presentan marcadas diferencias. Por ejemplo, dado que el promedio aproximado de autocontención es del $49 \%$, es evidente que hay marcadas desviaciones. Entre las zonas que superan el promedio, destaca la centrooriente, que presenta un $81 \%$ de autocontención, cifra que duplica con creces los porcentajes de las zonas sur y suroriente, que registran los valores más bajos.

En el cuadro 1 también queda en evidencia el carácter exógeno del empleo de los trabajadores residentes en todas las zonas, con excepción de la centrooriente, dado que la proporción de desplazamientos por razones de trabajo hacia esta es elevada. De hecho, un alto porcentaje de trabajadores de las dos zonas con mayor relación exógena entre el lugar de residencia y de trabajo se trasladan a la zona centrooriente, y en algunos casos este supera el de autocontención.

En cuanto a la porosidad, los datos presentados en el cuadro 1 revelan que esta es superior en la zona centrooriente, destino de numerosos desplazamientos originados en otras zonas, que superan los que se producen entre las restantes. Por otra parte, la suma de los porcentajes de autocontención y de traslados a la zona centrooriente permite concluir que en la mayoría de los casos la proporción de desplazamientos a esta por motivos de trabajo es superior al $75 \%$, pero que, en general, es baja en relación con las otras.

La frecuencia de desplazamientos por razones de trabajo entre zonas de mercado laboral demuestra que para los habitantes de la zona centrooriente - seguida en menor medida por la zona norte- este es básicamente endógeno, mientras que en las demás es más exógeno y se centra en la primera. Además, la zona centrooriente es la que presenta una mayor porosidad, ya que recibe un gran número de trabajadores externos. Por lo tanto, de manera similar a lo observado en el estudio de la zona metropolitana del Valle de México (Casado, 2012), si bien es posible distinguir distintas zonas de mercado laboral local, que en Santiago de Chile presentan una configuración no radial, la existencia de un área con una gran capacidad de concentración de empleos da origen a una estructura territorial urbana dependiente de un centro ampliado, como ocurre con la zona centrooriente. Sin embargo, 
en términos generales, la ciudad de Santiago es un amplio mercado laboral, que se distingue de las áreas que la rodean y de las ciudades cercanas, y que abarca zonas con un nivel de autocontención relativamente elevado.

\section{Análisis de las desigualdades socioeconómicas}

En las grandes ciudades de América Latina, caracterizadas por un alto grado de desigualdad socioeconómica, la delimitación de las zonas de mercado laboral puede realzar el valor del análisis de las desigualdades, por lo que a continuación se examina su validez desde esta perspectiva en el caso de Santiago.

La desigualdad en términos de ingresos, elemento fundamental de la caracterización de las desigualdades socioeconómicas, se observa claramente en la clasificación por quintiles de ingreso de las zonas de mercado de trabajo (véase el cuadro 2).

Cuadro 2

Santiago de Chile: clasificación de las zonas de mercado laboral según quintiles de ingresos de los trabajadores ${ }^{a}, 2013$

(En porcentajes)

\begin{tabular}{lrrrrr}
\hline & I & II & III & IV & V \\
\hline Centrooriente & 3 & 7 & 8 & 20 & 63 \\
\hline Poniente & 12 & 23 & 29 & 24 & 12 \\
\hline Sur & 13 & 17 & 26 & 27 & 18 \\
\hline Surponiente & 17 & 24 & 25 & 24 & 10 \\
\hline Norte & 15 & 25 & 29 & 23 & 8 \\
\hline Suroriente & 21 & 23 & 26 & 26 & 5 \\
\hline Total & 13 & 19 & 22 & 23 & 23 \\
\hline
\end{tabular}

Fuente: Encuesta de Caracterización Socioeconómica Nacional (CASEN), 2013.

a La distribución corresponde a los ingresos autónomos regionales (medidos según la nueva metodología de la encuesta).

La comparación entre las zonas demuestra que presentan marcadas diferencias, en especial, una mayor concentración del quintil superior en la zona centrooriente y del quintil inferior en la suroriente. La prevalencia del quintil más alto en la zona centrooriente va acompañada de un nivel general de ingreso bastante superior al observado en las demás. De hecho, esta presentaba una mediana de ingresos autónomos equivalente a 1.260 dólares mensuales en $2013^{9}$, mientras que en las otras la mediana fluctuaba entre 533 dólares en la zona poniente y una cifra del orden de los 478 dólares en las surponiente, norte y sur. A la vez, dentro de las zonas, incluso en la centrooriente, existen variaciones que no se reflejan adecuadamente en la mediana de ingresos, entre otras medidas. Tal como ocurre con el nivel de educación, sería difícil hacer distinciones territoriales de índole general exclusivamente sobre la base del ingreso. Esto valida la distinción por zonas de mercado de trabajo, que también es muy útil para el análisis territorial de la desigualdad de ingresos, como se observa en la comparación del coeficiente de Gini (véase el gráfico 1).

La distinción entre las seis zonas facilita la comprensión de la desigualdad de ingresos en Santiago. El coeficiente de Gini es mucho más elevado en la zona centrooriente, mientras que en la suroriente es relativamente bajo. Las demás zonas presentan una desigualdad inferior al promedio, por lo que, en gran medida, la del conjunto de la ciudad obedece a la notable heterogeneidad que se registra en la zona centrooriente.

9 Según tipo de cambio vigente en junio de 2015 (627 pesos chilenos por dólar). 


\section{Gráfico 1}

Santiago de Chile: coeficiente de Gini de los trabajadores en las zonas de mercado laboral, 2013

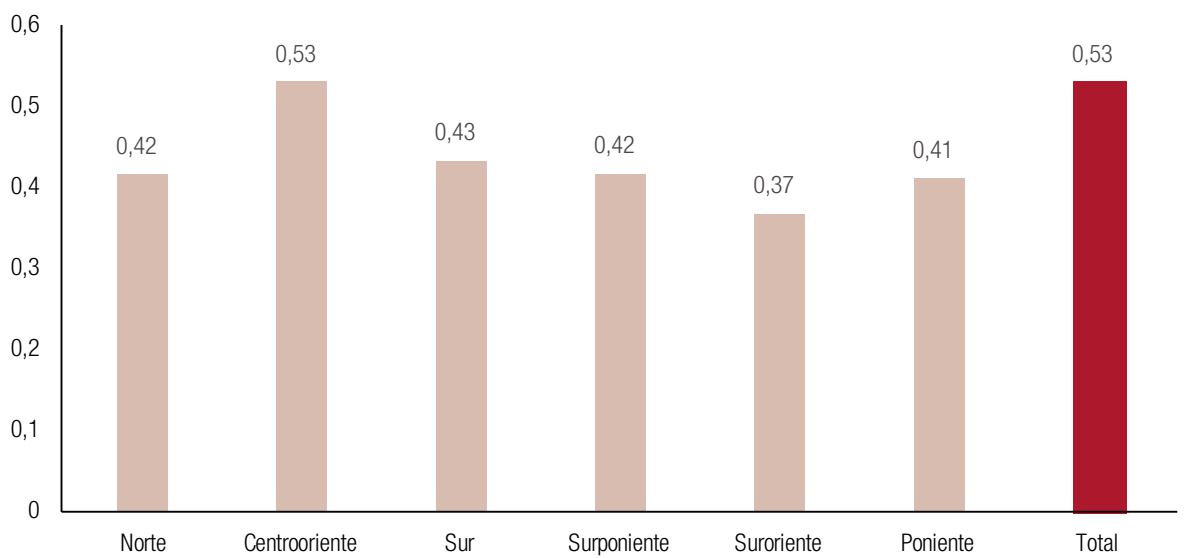

Fuente: Elaboración propia, sobre la base de Encuesta de Caracterización Socioeconómica Nacional (CASEN), 2013.

El nivel educacional es un factor clave de la desigualdad de ingresos y también muestra una heterogénea distribución en las distintas zonas de mercado laboral (véase el cuadro 3).

Cuadro 3

Santiago de Chile: nivel de educación de los residentes en las zonas de mercado laboral, 2013

(En porcentajes)

\begin{tabular}{lccc}
\hline & Básica & Media & Superior \\
\hline Centrooriente & 9 & 26 & 65 \\
\hline Poniente & 33 & 47 & 20 \\
\hline Sur & 31 & 44 & 25 \\
\hline Surponiente & 39 & 42 & 19 \\
\hline Norte & 39 & 45 & 16 \\
\hline Suroriente & 43 & 45 & 12 \\
\hline Total & 29 & 40 & 30 \\
\hline
\end{tabular}

Fuente: Encuesta de Caracterización Socioeconómica Nacional (CASEN), 2013.

La zona centrooriente no solo se caracteriza por tener el más alto nivel de ingreso de Santiago, sino también por contar entre sus habitantes con una alta proporción de profesionales y técnicos con estudios superiores; la zona surponiente le sigue en un segundo lugar, aunque con un porcentaje mucho menor. En cambio, una alta fracción de los trabajadores de las zonas suroriente, surponiente y norte solo tiene educación básica, completa o incompleta, o no tiene educación. Las zonas sur y poniente se encuentran en una situación intermedia.

La clara delimitación de las zonas que integran el espacio socioterritorial de la ciudad facilita la comprensión de las diferencias graduales en términos de ingreso y educación. Asimismo, el análisis basado en las zonas de mercado laboral permite entender mejor otras desigualdades territoriales en ámbitos tales como la vivienda. El valor aproximado de los bienes raíces se puede calcular sobre la base del Impuesto Territorial ${ }^{10}$. Según datos del Servicio de Impuestos Internos correspondientes

\footnotetext{
10 Se estima que el avalúo fiscal en el que se basa la determinación del Impuesto Territorial equivale aproximadamente a un 50\% del avalúo comercial. Los bienes raíces no agrícolas de uso residencial cuyo avalúo es inferior a unos 3.500 dólares (según tipo de cambio de junio de 2015) no están afectos a este impuesto.
} 
a 2014, en la zona centrooriente un 90\% de las propiedades está afecto al pago de este tributo, mientras que en las demás zonas el porcentaje es inferior y fluctúa entre un $25 \%$ en la norte y un $8 \%$ en la suroriente; las propiedades restantes están exentas de pago. Cabe señalar que, en promedio, en el área estudiada el 31\% de las propiedades están afectas al Impuesto Territorial; este porcentaje se triplica en la zona centrooriente, seguida por la zona sur, en la que es levemente superior al promedio. Estas acentuadas diferencias reflejan el valor de los bienes raíces en cada una de las zonas y, por lo tanto, dan cuenta de la desigualdad en materia de activos inmobiliarios. El avalúo fiscal promedio varía considerablemente de una zona a otra; en el sector centrooriente es 1,5 veces mayor que el promedio de la ciudad y 3,5 veces superior al de la zona suroriente, que presenta el avalúo promedio más bajo de todas.

\section{Composición de las zonas por clases sociales}

La composición de las zonas de mercado laboral por clases sociales explica en gran medida las diferencias que se dan en este ámbito tanto entre zonas como en cada una de ellas. Las clases sociales son grupos numerosos, definidos en general por el lugar que ocupan en la jerarquía social y por la relación con otros conglomerados en términos de posesión de bienes de uso o productivos, prestigio y otros activos sociales o simbólicos (Mac-Clure, Barozet y Maturana, 2014). Para analizar la estructura social de las zonas consideradas, se aplica la clasificación EGP (Erikson y Goldthorpe, 1993 $)^{11}$, una de las más utilizadas a nivel internacional y en varios estudios realizados en Chile (Espinoza, Barozet y Méndez, 2013; Mac-Clure, 2012; Mac-Clure, Barozet y Maturana, 2014; Torche y Wormald, 2004). En esta clasificación se aplican criterios relativos a la propiedad y el control de los medios productivos, la autonomía, el tipo de actividad de los trabajadores (manual o no manual) y su calificación. En el cuadro 4 se indica la composición por clases sociales de las zonas de mercado laboral de la ciudad de Santiago.

Cuadro 4

Santiago de Chile: clases sociales por zonas de mercado laboral, 2013

(En porcentajes)

\begin{tabular}{|c|c|c|c|c|c|c|c|}
\hline \multirow{2}{*}{ Clase } & \multicolumn{6}{|c|}{ Zona } & \multirow[b]{2}{*}{ Total } \\
\hline & Centrooriente & Poniente & Sur & Surponiente & Norte & Suroriente & \\
\hline Servicios de alta categoría & 29 & 6 & 8 & 6 & 5 & 2 & 11 \\
\hline Servicios de baja categoría & 30 & 10 & 15 & 10 & 10 & 8 & 16 \\
\hline $\begin{array}{l}\text { Ocupaciones rutinarias no } \\
\text { manuales de alta categoría }\end{array}$ & 9 & 12 & 11 & 11 & 11 & 12 & 11 \\
\hline $\begin{array}{l}\text { Ocupaciones rutinarias no } \\
\text { manuales de baja categoría }\end{array}$ & 9 & 15 & 15 & 13 & 12 & 13 & 12 \\
\hline Pequeños empresarios & 1 & 1 & 1 & 1 & 1 & 1 & 1 \\
\hline Trabajadores independientes & 9 & 16 & 16 & 18 & 18 & 18 & 15 \\
\hline Trabajadores manuales calificados & 4 & 15 & 11 & 17 & 14 & 17 & 12 \\
\hline Trabajadores manuales no calificados & 10 & 25 & 23 & 23 & 29 & 28 & 22 \\
\hline Trabajadores agrícolas & 0 & 1 & 1 & 1 & 2 & 1 & 1 \\
\hline Agricultores & 0 & 0 & 0 & 1 & 1 & 0 & 0 \\
\hline
\end{tabular}

Fuente: Elaboración propia, sobre la base de Encuesta de Caracterización Socioeconómica Nacional (CASEN), 2013.

\footnotetext{
${ }^{11}$ La variable "clases sociales", basada en el esquema EGP aplicado a Chile, se obtuvo de las encuestas CASEN utilizando para ello una sintaxis elaborada por Vicente Espinoza, a quien agradecemos su contribución.
} 
Como se observa en el cuadro 4, la composición de las clases sociales en Santiago se caracteriza por una participación predominante de los trabajadores manuales no calificados. Con una proporción menor le siguen la clase de servicios de baja categoría, compuesta principalmente por técnicos; los trabajadores independientes, incluidos los trabajadores por cuenta propia no profesionales; la clase de ocupaciones rutinarias no manuales de baja categoría, integrada por asalariados que se desempeñan como vendedores o prestadores de servicios, y los trabajadores manuales calificados. El tercer grupo de clases sociales, que presenta porcentajes levemente inferiores, corresponde a la clase de servicios de alta categoría, constituida por profesionales con título universitario, y a la clase de ocupaciones rutinarias no manuales de alta categoría, de la que forman parte los empleados administrativos de nivel intermedio. Finalmente, las clases con menor participación son las de pequeños empresarios, trabajadores agrícolas y agricultores. En esta categorización de las clases sociales presentes en Santiago no se incluye a la élite socioeconómica, debido a que generalmente no está representada en las encuestas de hogares y a que concentra gran parte del poder social, económico y político del país. La clase de servicios de alta categoría, descrita anteriormente, ocupa las posiciones más cercanas a la élite.

Si se considera la distribución territorial de las clases en las distintas zonas, los porcentajes varían. En este contexto, destaca la zona centrooriente, en la que están sobrerrepresentadas las clases de servicios de alta y baja categoría. La concentración de integrantes de la primera en esta zona concuerda con la elevada desigualdad de ingresos que presenta, porque estos difieren notablemente de los correspondientes a las demás clases sociales y, además, muestran una gran dispersión interna. La clase de servicios también está representada en la zona sur aunque en menor medida, y especialmente por técnicos y profesionales de nivel intermedio. En esta zona, al igual que en la poniente, hay un alto porcentaje de empleados administrativos y vendedores de la clase de ocupaciones rutinarias de baja categoría. En las zonas poniente, surponiente, norte y suroriente se observa una elevada participación de los trabajadores manuales, que en las dos últimas son en su mayoría no calificados. A diferencia de la zona centrooriente, en estas cuatro la clase de servicios en general está subrepresentada.

A largo plazo y desde el punto de vista de la movilidad social intergeneracional, dentro de cada zona las clases sociales se diferencian en cuanto al origen de sus integrantes, la educación de los padres y una variable que habitualmente no se toma en consideración en los estudios: el lugar de nacimiento. Los miembros de la clase de servicios de alta categoría que viven en la zona centrooriente, en la que son más numerosos que en las demás, provienen de familias en las que, en gran parte de los casos, el padre no cursó estudios superiores y nació en una comuna de otra zona. Por consiguiente, la zona centrooriente es un mercado laboral en el que los estratos superiores de la clase media son producto de una intensa movilidad social y una notable migración territorial intergeneracional.

En el período comprendido entre 1996 y 2013, se produjo un acelerado incremento de la proporción del total de trabajadores residentes en la zona centrooriente correspondiente a las clases de servicios, unido a una disminución de los trabajadores manuales. Esto último obedece, entre otras cosas, a que frecuentemente los hijos de los habitantes de comunas de esta zona no pueden optar por seguir residiendo en ellas debido al encarecimiento de los suelos, que se traduce en un aumento del precio de las unidades habitacionales, y a la construcción de viviendas sociales en comunas periféricas de la ciudad, donde el precio es menor. En contraste con lo que ocurre en la zona centrooriente, en las demás la participación de las clases de ocupaciones rutinarias no manuales de alta y baja categoría y de trabajadores manuales calificados y no calificados se mantiene invariable. Todo esto indica que el proceso de movilidad social ascendente se concentra en dicha zona.

Presumiblemente, las múltiples desigualdades existentes en las zonas de mercado laboral obedecen a las diferencias de carácter social. Además, la presencia de las diversas clases sociales en estas zonas permite entender mejor variadas preferencias y percepciones subjetivas. 
La heterogeneidad de la ciudad en materia de equipamiento, que influye en la calidad de vida de sus habitantes (Orellana, 2014), contribuye a la migración hacia determinadas zonas. La centrooriente, que presenta un alto nivel de concentración de activos fijos, absorbió el 40\% de las autorizaciones de edificación y el 68\% de las construcciones realizadas en la Región Metropolitana entre 2000 y 2012 (datos sobre edificación del Instituto Nacional de Estadísticas). Esta mayor dotación de activos fijos - a la que se suman mejores servicios, entre otros, de salud, educación y seguridad- explicaría, desde el punto de vista del interés económico, tanto la preferencia de los integrantes de las clases de servicios de alta y baja categoría por residir en la zona centrooriente como la estrategia de las empresas inmobiliarias, orientada selectivamente a esta zona. La forma en que opera el mercado inmobiliario facilita la movilidad residencial ascendente (Sabatini, 2006), en particular la de los integrantes de dichas clases, que emigran a la zona centrooriente.

La preferencia por un área de residencia u otra (Sabatini y otros, 2012) también responde a motivaciones subjetivas, entre otras las de los estratos superiores de las clases medias que viven en la zona centrooriente, relacionadas con el interés por incrementar su capital cultural y social mediante mecanismos de distinción social (Bourdieu, 2012). Los residentes en las restantes zonas -y los menos favorecidos de la zona centrooriente- deben recurrir a otros medios para mantener o mejorar su condición social. De acuerdo con estudios recientes basados en metodologías inspiradas en juegos (Barozet y Mac-Clure, 2014; Mac-Clure, Barozet y Moya, 2015), desde un punto de vista subjetivo, los integrantes de las capas menos privilegiadas de las clases medias consideran que hay tres factores determinantes del incremento del ingreso: el esfuerzo personal, que se impone al limitado acceso a recursos económicos preexistentes; el desarrollo del capital cultural, asociado a la educación, y el capital social, vinculado a la familia y al círculo más cercano. En particular, atribuyen una importancia fundamental al esfuerzo educativo y laboral, que a su juicio es más relevante que el capital económico acumulado o heredado, del que carecen, y el valor simbólico y los recursos que suponen los activos fijos como los existentes en la zona centrooriente, junto con criticar las "discriminaciones" asociadas a la comuna de residencia original.

Para resumir la caracterización socioeconómica de las zonas de mercado laboral de la ciudad de Santiago derivada del análisis efectuado, la zona centrooriente está habitada principalmente por un estrato medio-alto con ingresos elevados, pero también se caracteriza por la marcada desigualdad económica de sus residentes. La mayor parte de estos tienen educación superior universitaria o técnica $y$, desde el punto de vista ocupacional, pertenecen a las clases de servicios, integradas especialmente por profesionales de la clase de servicios de alta categoría, cuya preferencia por esta zona refleja el valor que le otorgan como símbolo de movilidad social ascendente. Los habitantes de las zonas poniente y sur pertenecen al estrato medio, en el que predominan los empleados y vendedores de la clase de ocupaciones rutinarias no manuales, de ingreso medio-bajo; en estas la desigualdad interna es moderada en comparación con la zona centrooriente, y se observa una aspiración a la movilidad social basada en el esfuerzo laboral y educativo. Sin embargo, ambas presentan peculiaridades que las diferencian: la mayor parte de los habitantes de la zona poniente tienen educación media, mientras que en la zona sur hay una proporción levemente más alta de personas con educación superior; en esta última, también hay un mayor porcentaje de técnicos y profesionales de nivel intermedio pertenecientes a la clase de servicios de baja categoría.

En términos de jerarquías sociales, en las zonas surponiente, norte y suroriente, en las que reside más de la mitad de los habitantes de la ciudad, predominan los integrantes de la clase de trabajadores manuales. En estas zonas el ingreso de la población es bajo y la desigualdad económica es moderada, incluso relativamente reducida en la suroriente. El nivel educacional es inferior al de las zonas habitadas mayoritariamente por miembros de las clases medias, pero mientras que en las zonas surponiente y norte predomina la población con educación media, en la suroriente hay una alta proporción de residentes que solo tienen educación básica. Si bien las zonas surponiente y norte 
presentan similitudes, en la primera hay una mayor proporción de trabajadores manuales calificados que en la segunda, en la que se observa un claro predominio de la clase de trabajadores manuales no calificados. En general, la condición socioeconómica de estos no varía, lo que contrasta con la movilidad ascendente que se da en las zonas de residencia de las clases medias, especialmente la centrooriente.

\section{Conclusiones}

En síntesis, en el estudio se demuestra la pertinencia de un enfoque metodológico basado en el análisis de los mercados laborales locales, en el que se considera el traslado entre comunas, para el análisis de grandes ciudades como Santiago de Chile. Los criterios y procedimientos metodológicos descritos permiten concluir que en el área metropolitana de Santiago pueden distinguirse seis zonas en función de los mercados laborales.

De acuerdo con los indicadores socioeconómicos analizados, las mayores diferencias que se observan en la ciudad se producen entre la zona centrooriente y los demás mercados laborales. Asimismo, se determinó que dicha zona presenta las desigualdades sociales más marcadas, que se manifiestan, entre otras cosas, en un elevado coeficiente de Gini. En la zona centrooriente conviven numerosos integrantes de los dos extremos de la jerarquía social: la clase de servicios de alta categoría y la clase de trabajadores manuales no calificados. La primera de ellas es predominante, por lo que en términos de categorías sociales esta zona es la más polarizada de todas. Por consiguiente, la desigualdad social en la ciudad de Santiago es de carácter eminentemente espacial, y obedece fundamentalmente a la heterogeneidad de la zona centrooriente, y a las diferencias entre esta y las demás zonas de mercado laboral de la ciudad. Las desigualdades sociales existentes en las restantes zonas son más moderadas y están determinadas por la alta proporción de integrantes de la clase de trabajadores manuales que residen en ellas, con la excepción de la zona surponiente, en la que hay una mayor presencia de los estratos medios correspondientes a la clases de servicios de baja categoría y de ocupaciones rutinarias no manuales de alta y baja categoría.

Las conclusiones expuestas confirman la relevancia de la aplicación de un enfoque territorial basado primordialmente en la configuración de zonas de mercado laboral, para el estudio de las desigualdades sociales en un área metropolitana. Los mercados de trabajo condicionan las desigualdades geográficas en la ciudad de Santiago, que se expresan espacialmente en diferencias sustantivas entre las zonas con respecto al ingreso, el acceso a la educación y la composición de las clases sociales. Entre las zonas operan también mecanismos de distinción social y factores subjetivos vinculados al capital cultural y social. En el caso de Santiago, el carácter espacial de las desigualdades se manifiesta doblemente, tanto entre el espacio metropolitano en su conjunto y el resto del país como dentro de la ciudad, integrada por diversas zonas de mercado laboral local. Esta conclusión facilita la comprensión de los espacios urbanos, dado que el análisis realizado demuestra que las zonas de mercado laboral se caracterizan por una configuración socioeconómica y de clases sociales, y no son solo un fenómeno sociodemográfico exclusivamente relacionado con el lugar de residencia y el traslado por razones de trabajo.

El presente estudio aporta elementos concretos que contribuyen a la comprensión de la ciudad de Santiago, especialmente en lo que se refiere a aspectos poco estudiados de la conformación y transformación de la estructura socioterritorial. Las marcadas diferencias entre las zonas de mercado laboral permiten confirmar que Santiago es una ciudad en la que, al parecer, coexisten varias ciudades. En cierta medida, las diferencias sociales entre distintas áreas de las metrópolis de América Latina y de otras regiones son habituales; lo nuevo del análisis presentado en este artículo es la aplicación de un método de delimitación y descripción de esos espacios urbanos, a partir de las cuales se observa 
que la trayectoria de las clases sociales en las zonas de mercado laboral de Santiago registrada en la última década tiende a acentuar y consolidar las diferencias sociales que presenta la ciudad, en lugar de reducirlas. La consideración de las peculiaridades de estas zonas nos permite aplicar el concepto "ciudad de ciudades", utilizado por Nel.Lo (2002) para referirse a Barcelona, y ampliarlo desde una perspectiva vinculada a la configuración socioespacial de una gran ciudad como Santiago.

\section{Bibliografía}

Agostini, C.A. (2010), "Pobreza, desigualdad y segregación en la Región Metropolitana”, Estudios Públicos, № 117, Santiago, Centro de Estudios Públicos (CEP).

Arriagada, C. (2010), "Segregación residencial según dos modelos de urbanización y bienestar: estudio comparado de las áreas metropolitanas del Gran Santiago, Toronto y Vancouver", Notas de Población, vol. 37, № 91 (LC/G.2484-P), Santiago, Comisión Económica para América Latina y el Caribe (CEPAL).

Barozet, E. y O. Mac-Clure (2014), "Nombrar y clasificar: aproximación a una epistemología de las clases sociales", Cinta de Moebio. Revista de Epistemología de Ciencias Sociales, № 51, Santiago, Facultad de Ciencias Sociales, Universidad de Chile.

Berdegué, J. y otros (2011) "Territorios funcionales en Chile", Documento de Trabajo, № 102, Santiago, Centro Latinoamericano para el Desarrollo Rural.

Bourdieu, P. (2012), La distinción. Criterio y bases sociales del gusto, Madrid, Taurus.

Casado, J.M. (2012), "La estructura policéntrica de los mercados laborales locales de la Zona Metropolitana del Valle de México", Investigaciones Geográficas, № 79, Ciudad de México, Universidad Nacional Autónoma de México (UNAM).

Casado-Díaz, J. (2003), "The use of commuting data to define local labour market areas and urban areas in Spain", documento presentado en la NECTAR Conference "A New Millenium. Are Things The Same? Innovation, Sustainability and Efficiency in Communication and Transportation Systems", Umeå, Suecia, Universidad de Umeå.

Casado-Díaz, J. y M. Coombes (2011), "The delineation of 21st century local labour market areas: a critical review and a research agenda", Boletín de la Asociación de Geógrafos Españoles, № 57.

Cattaneo, R. (2012), La fabrique de la ville: promoteurs immobiliers et financiarisation de la filière du logement à Santiago du Chili, París, Université Paris 8.

Coombes, M. y S. Bond (2008), Travel-to-Work Areas: the 2007 Review, Londres, Oficina Nacional de Estadística.

De Mattos, C. (2010), Globalización y metamorfosis urbana en América Latina, Quito, Organización Latinoamericana y del Caribe de Centros Históricos (OLACCHI).

- (2002), "Mercado metropolitano de trabajo y desigualdades sociales en el Gran Santiago: ¿Una ciudad dual?", EURE, vol. 28, № 85, Santiago, Pontificia Universidad Católica de Chile.

De Mattos, C., L. Fuentes y F. Link (2014), "Tendencias recientes del crecimiento metropolitano en Santiago de Chile. ¿Hacia una nueva geografía urbana?”, Revista INVI, vol. 29, № 81, Santiago, Facultad de Arquitectura y Urbanismo, Universidad de Chile.

Dockendorff, E. y otros (1990), Santiago, dos ciudades: análisis de la estructura socio-económica espacial del Gran Santiago, Santiago, Centro de Estudios del Desarrollo (CED).

Erikson, R. y J.H. Goldthorpe (1993), The Constant Flux, Oxford, Oxford University Press.

Escolano, S. y J. Ortiz (2005), "La formación de un modelo policéntrico de la actividad comercial en el Gran Santiago (Chile)", Revista de Geografía Norte Grande, №34, Santiago, Pontificia Universidad Católica de Chile.

Espinoza, V., E. Barozet y M.L. Méndez (2013), "Estratificación y movilidad social bajo un modelo neoliberal: El caso de Chile", Lavboratorio, № 25, Buenos Aires, Instituto Gino Germani/Universidad de Buenos Aires.

Heinrichs, D. y otros (eds.) (2011), Risk Habitat Megacity, Heidelberg, Springer.

ISTAT (Instituto Nacional de Estadística) (2005), I sistema locali del lavoro. Censimento 2001. Dati definitivi [en línea] http://dawinci.istat.it/daWinci/jsp/MD/download/sll_comunic_solo_testo.pdf.

Mac-Clure, O. (2012), "Las nuevas clases medias en Chile: un análisis de cohortes", Revista CEPAL, № 108 (LC/G.2549-P), Santiago, Comisión Económica para América Latina y el Caribe (CEPAL).

Mac-Clure, O. y R. Calvo (2013), "Desigualdades sociales y tipos de territorios en Chile", Polis, № 34, Osorno, Chile, Universidad de Los Lagos. 
Mac-Clure, O., E. Barozet y V. Maturana (2014), "Desigualdad, clase media y territorio en Chile: ¿clase media global o múltiples mesocracias según territorios?", EURE, Santiago, vol. 40, № 121, Santiago, Pontificia Universidad Católica de Chile.

Mac-Clure, O., Barozet, E. y C. Moya (2015), “Juicios de las clases medias sobre la élite económica: ¿crítica a las desigualdades en Chile?", Polis, № 41, Osorno, Chile, Universidad de Los Lagos.

Maturana, F. y F. Arenas (2012), "El policentrismo en Chile: medición exploratoria para el sistema de ciudades de las regiones de La Araucanía, de Los Ríos y de Los Lagos", Revista de Geografía Norte Grande, Nㅜ 52 , Santiago, Pontificia Universidad Católica de Chile.

Nel.Lo, O. (2002), Cataluña, ciudad de ciudades, Lleida, Editorial Milenio.

OCDE (Organización para la Cooperación y el Desarrollo Económicos) (2002), Redefining Territories: Functional Regions, París, OECD Publishing.

Orellana, A. (2014), Indicador de calidad de vida ciudades chilenas (ICVU), Santiago, Estudios Urbanos UC/ Cámara Chilena de la Construcción.

(2009), "La gobernabilidad metropolitana de Santiago: la dispar relación de poder de los municipios", EURE, vol. 35, № 104, Santiago, Pontificia Universidad Católica de Chile.

Orellana, A. y L. Fuentes (2007), "El arco, el cono y el cluster: geometrías espaciales para la gobernabilidad metropolitana y local de Santiago", Ciudad, poder y gobernanza, G. Yáñez y otros (eds.), Santiago, Colección Rideal, Eure Libros y Geolibros.

Ortiz, J. y S. Escolano (2013), "Movilidad residencial del sector de renta alta del Gran Santiago (Chile): hacia el aumento de la complejidad de los patrones socioespaciales de segregación", EURE, vol. 39, № 118, Santiago, Pontificia Universidad Católica de Chile.

Ortiz, J. y S. Morales (2002), "Impacto socioespacial de las migraciones intraurbanas en entidades de centro y de nuevas periferias del Gran Santiago", EURE, vol. 28, № 85, Santiago, Pontificia Universidad Católica de Chile.

Pollack, M. y A. Uthoff (1987), "Pobreza y mercado de trabajo en el Gran Santiago: 1969-1985", Documento de Trabajo, № 299, Santiago de Chile, Programa Regional del Empleo para América Latina y el Caribe (PREALC).

Riffo, L. (2004), "Los impactos de la globalización sobre los mercados de trabajo metropolitanos: el caso de Santiago de Chile en la década de los noventa", Santiago en la globalización ¿una nueva ciudad?, C. de Mattos y otros (eds.), Santiago, Ediciones SUR.

Rodríguez, J. (2012), “¿Policentrismo o ampliación de la centralidad histórica en el Área Metropolitana del Gran Santiago? Evidencia novedosa proveniente de la encuesta Casen 2009", EURE, vol. 38, № 114, Santiago, Pontificia Universidad Católica de Chile.

Russo, G. y otros (2011), "Commuter effects on local labour markets: a German modelling study", Tinbergen Institute Discussion Papers, № 11-114/3, Tinbergen Institute.

Sabatini, F. (2006), "La segregación social del espacio en las ciudades de América Latina", Working Paper, Washington, D.C., Banco Interamericano de Desarrollo.

Sabatini, F., G. Cáceres, y J. Cerda (2001), "Segregación residencial en las principales ciudades chilenas: Tendencias de las tres últimas décadas y posibles cursos de acción”, EURE, vol. 27, № 82, Santiago, Pontificia Universidad Católica de Chile.

Sabatini, F. y otros (2012), “¿Es posible la integración residencial en las ciudades chilenas? Disposición de los grupos medios y altos a la integración con grupos de extracción popular", EURE, vol. 38, № 115, Santiago, Pontificia Universidad Católica de Chile.

Sassen, S. (2001), The Global City, Princeton, Princeton University Press.

SECTRA (Secretaría de Planificación de Transporte) (1992), Estudio encuesta origen destino de viajes del Gran Santiago, 1991, Santiago.

Singelmann, J. y F. Deseran (eds.) (1993), Inequalities in Labor Market Areas, Boulder, Colorado, Westview Press.

Tolbert, C. y M. Sizer (1996), "U.S. commuting zones and labor market areas. A 1990 update", Staff Paper, $N^{\circ}$ AGES-9614, Washington, D.C., Departamento de Agricultura de los Estados Unidos.

Torche, F. y G. Wormald (2004), "Estratificación y movilidad social en Chile: entre la adscripción y el logro", Serie Políticas Sociales, № 98 (LC/L.2209-P), Santiago, Comisión Económica para América Latina y el Caribe (CEPAL).

Truffello, R. y R. Hidalgo (2015), "Policentrismo en el Área Metropolitana de Santiago de Chile: reestructuración comercial, movilidad y tipificación de subcentros", EURE, vol. 41, № 122, Santiago, Pontificia Universidad Católica de Chile.

Vallès, V. (2004), "Bassins de vie: au centre de la vie quotidienne", La Lettre, № 14, París, Instituto Nacional de Estadística y Estudios Económicos (INSEE). 